\title{
Sanidad y la II República Española (1931-1936): la creación de la figura de la primera Directora de Enfermeras
}

\section{Health and the Second Spanish Republic (1931-1936): the creation of the figure of the first Head of Nurses}

\section{Saúde e da Segunda República Espanhola (1931-1936): a criação da figura do primeiro Director of Nurses}

\author{
Francisco-Javier Castro-Molina ${ }^{1-2}$, Natalia Rodríguez-Novo², Felisa-Vanessa Martín-Casañas ${ }^{2}$ \\ ${ }^{1}$ Escuela Universitaria de Enfermería de "Nuestra Señora de Candelaria" (adscrita a la Universidad de La Laguna-ULL). \\ ${ }^{2}$ Asociación Canaria de Historia de la Profesión Enfermera (ACHPE).
}

Cómo citar este artículo en edición digital: Castro-Molina, F.J., Rodríguez-Novo, N., \& Martín-Casañas, F.V. (2017). Sanidad y la II República Española (1931-1936): la creación de la figura de la primera Directora de Enfermeras. Cultura de los Cuidados (Edición digital), 21(48). Recuperado de < http://dx.doi.org/10.14198/cuid.2017.48.10>

Correspondencia: Asociación Canaria de Historia de la Profesión Enfermera (ACHPE), calle San Martín 62, 38001-Santa Cruz de Tenerife.

Correo electrónico: fjcastrom7@gmail.com

Recibido: 08/12//2016; Aceptado: 03/03/2017

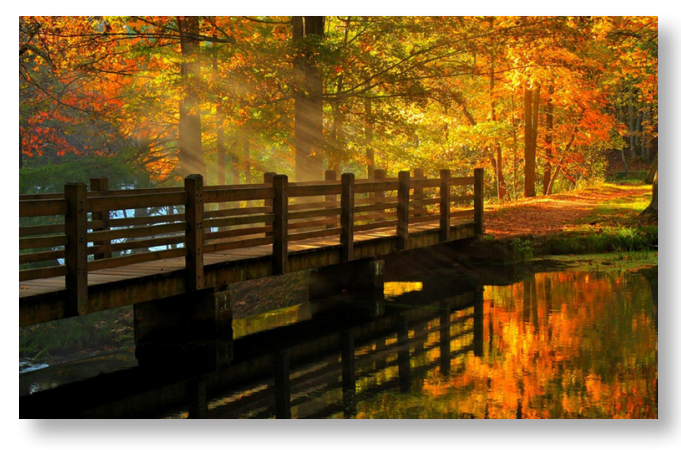

ABSTRACT

Introduction: Healthcare in Spain, as in many other countries, was a top concern for their ruler. But it is during the Second Spanish Republic (1931-1936) when this restlessness becomes more alive. To improve it was allocated a budget that, after exercise, was increased to solve the serious problems that suffered, seeking only the modernization of existing infrastructures and the creation of so many new ones, in addition to achieving a maximum qualification of its professionals. This last one was of considerable draft, matching it with the flamboyant examples of many other European countries that bet on leaving behind models anchored in the Middle Ages and giving way to a system that functions as a "healing machine".

Objective: Establish when the figure of the Director of Nurses emerges for the first time in Spain.

Method: A "thorough examination" of the documentation was carried out in the Archive of the Very Insular Cabildo of Santa Cruz de Tenerife and bibliographically.

Results: In the Canary Islands, as in many other places in the "bullskin", the reforms were pursued deep. A clear example of this was the regulation, of short time in force, formulated by the councilor of the Insular Cabildo of Tenerife Miguel Bethencourt of the River, that gave a turn to the existing organization until that moment.

Conclusions: Among the changes highlighted the creation of the figure of 
Director of Nurses, unpublished until now, and that we had to wait for the sunset of the Franco dictatorship to see it again in the Spanish Health.

Keywords: History, nursing, director of nurses, management, canary islands, II Spanish Republic.

\section{RESUMEN}

Introducción: La sanidad en España, como en otros tantos países, fue una preocupación máxima para sus gobernante. Pero es durante la Segunda República Española (1931-1936) cuando esta inquietud se hace más viva. Para su mejora se destinó un presupuesto que, ejercicio tras ejercicio, fue aumentando para solventar los graves problemas que sufría, buscando tan solo la modernización de las infraestructuras existentes y la creación de otras tantas nuevas, además de lograr una máxima cualificación de sus profesionales. Esta última fue de considerable calado equiparándola con los flamantes ejemplos de otros muchos países europeos que apostaban por dejar atrás modelos anclados en el medievo $\mathrm{y}$ dar paso a un sistema que funcionara como una "máquina de sanación".

Objetivo: Establecer cuando surge por primera vez en España la figura de la Directora de Enfermeras.

Método: Se realizó un "examen exhaustivo" de la documentación custodiados en el Archivo del Excelentísimo Cabildo Insular de Santa Cruz de Tenerife y bibliográficamente.

Resultados: En Canarias, como en otros tantos lugares de la "piel de toro", las reformas se procuraron profundas. Un claro ejemplo de ello fue el reglamento, de corto tiempo en vigor, formulado por el consejero del Cabildo Insular de Tenerife Miguel Bethencourt del Rio, que daba un giro a la organización exis- tente hasta ese momento.

Conclusiones: Entre los cambios destacó la creación de la figura de Directora de Enfermeras, inédita hasta la fecha, y que hubo que esperar al ocaso de la Dictadura Franquista para verla nuevamente en la Sanidad Española.

Palabras clave: Historia, enfermería, directora de enfermeras, gestión, canarias, II República Española.

\section{RESUMO}

Introdução: Saúde em Espanha, como em muitos outros países, era uma das principais preocupações para a sua régua. Mas é durante a Segunda República espanhola (1931-1936), quando esta preocupação torna-se mais viva. Relativamente ao orçamento de melhoria que ano após ano, foi aumentando para resolver os graves problemas sofridos, procurando apenas a modernização da infra-estrutura existente e criar outro era para muitos nova adição para atingir profissionais máximos qualificados. $\mathrm{O}$ último foi de considerável profundidade equiparando-a com os novos exemplos de muitos outros países europeus a deixar para trás padrões de apostas ancoradas na Idade Média e dar lugar a um sistema que funcionaria como uma "máquina de cura".

Objectivo: Definir quando a figura do director de Enfermeiros surge pela primeira vez em Espanha.

Método: uma "revisão completa" dos documentos mantidos no Arquivo de Sua Excelência Conselho de Ilha de Santa Cruz de Tenerife e bibliograficamente foi realizada.

Resultados: Nas Ilhas Canárias, como em outros lugares da "pele de touro" reformas profundas procurado. Um exemplo claro disso foi a regulamentação em vigor curto espaço de tempo, feita pelo diretor do Cabildo Insular de Tenerife Bethencourt Miguel del Rio, que 
deu um toque para a organização existente até agora.

Conclusões: Entre as mudanças, ele destacou a criação do cargo de Diretor de Enfermeiros, inéditos até à data, e não foi até o crepúsculo do franquista para vê-lo novamente no Saúde espanhol.

Palavras-chave: História, enfermagem, eirector dos enfermeiros, gestão, canarias, II República Espanhola.

\section{INTRODUCCIÓN}

Uno de los momentos claves para la Sanidad Española fue el que se desarrolló durante el gobierno de la Segunda República, cuya gestión estuvo definida por un marcado intento de modernización de España. Tras la abdicación de Alfonso XIII se procedió a la proclamación del gobierno republicano, breve periodo que se vio truncado por el alzamiento militar liderado por Francisco Franco y que concluyó con el fatal desenlace de la Guerra Civil. Caracterizado este momento político por un desarrollo poco uniforme, es durante el gobierno del Frente Popular cuando se busca concluir las reformas que el régimen de Primo de Rivera habían puesto en funcionamiento (CastroMolina, 2013). Centrada básicamente en la creación de infraestructuras sanitarias acordes a los tiempos y en una organización sin precedentes de los recursos, su política se pivotó en torno a una activa voluntad preventiva, en el que la figura de la Enfermera se consolidó como "profesional de los cuidados y de la prevención" dentro de un equipo que comenzaba a mutar hacia el modelo multidisciplinar. Estos avances en el campo sanitario y social fueron adaptados al "ideario del Franquismo", reinterpretándolos y adjudicándoselos como propios, y siempre con un control férreo desde los órganos centrales de gobierno (Matesanz, 2009). Como un remedo de lo que acaecía en el resto del territorio nacional, en Canarias se apostó por una renovación de la red asistencial existente, en la que jugó un papel destacado la “desacralización” del espacio sanitario, apoyada en el cientificismo imperante tras la Revolución Industrial. Dejando atrás los nosocomios fundamentados en la filosofía escolástica y los preceptos galénicos, se abrió el camino hacia el hospital como "Máquina de Sanación”, donde se efectuó un giro copernicano tanto en su continente como en su contenido. En este, en su contenido, es donde surge una primacía, la Directora de Enfermeras, figura desconocida hasta la fecha que no distó en atribuciones, estatus y gobernanza a las que en la actualidad acampan en los diferentes hospitales que están diseminados por la red asistencial española (Herrera, 2003).

\section{MATERIAL Y MÉTODO}

El proceso de investigación del presente estudio constó de tres fases: primeramente se realizó un "examen exhaustivo" de la documentación custodiados en el Archivo del Excelentísimo Cabildo Insular de Santa Cruz de Tenerife, Sección de Beneficencia (expedientes de las cajas 6544, 6545, 6546, 7088). Posteriormente se procedió a una revisión bibliográfica de las publicaciones existentes en lengua española desde tres ópticas diferentes claramente definidas: la socio-histórica, la sanitaria y la antropológica (tesis doctorales y libros publicados presentes en la bibliografía). Y finalmente, se realizó una búsqueda en los diferentes recursos electrónico y bases de datos (CUIDEN, SCIELO y PubMed) atendiendo a las palabras Historia, Ciencias de la Salud, Enfermería, Directora de Enfermeras, gestión sanitaria y II República Española. 


\section{RESULTADOS}

El primer tercio del siglo XX en España constituyó una de los tramos históricos en los que "el intento de cambio" fue una constante. Desarrollado entre las dos dictaduras que estuvieron presentes en este siglo, la dirigida por Miguel Primo de Rivera (1923-1930) y la personificada por Francisco Franco (19391975), el momento de la II República Española constituyó un experimento político que nos colocó, constitucionalmente, por delante de numerosas naciones democráticas europeas. La finalidad de la propuesta republicana tenía como única meta la modernización de un país anclado en un modelo sociopolítico medieval. Tras la abdicación de Alfonso XIII y la proclamación del "representativo y popular gobierno rojo" su acción intensa se centró en buscar la homogeneidad de la población española mediantes políticas que igualaran los derechos. Durante el "Bienio Reformista", comprendido entre 1931 y 1933, las acciones renovadoras fueron tibias y poco uniformes, constituyendo una continuación de las iniciadas por Primo de Ribera. Inicialmente tres fueron los pilares en los que se basaron: búsqueda de una descentralización del Estado, gestionado por gobiernos autonómicos; la reforma agraria, con las expropiaciones forzosas y la instalación de comunidades campesinas; y por último, la reforma religiosa, que comprendía la laicidad del Estado (separación Iglesia-Estado), el sustento propio de ésta y la instauración del matrimonio civil y el divorcio. A ellas se le unieron cambios en otras áreas como la política, la educativa y la sanitaria (Huertas, 2000). La primera centró su interés en la instauración del voto femenino, mientras que la segunda, la reforma educativa, trajo como novedoso una educación obligatoria, pública y gratuita, ajena al mundo eclesiástico. La última, buscó reorganizar las infraestructuras sanitarias existentes, así como la creación de nuevos recursos sanitarias con una activa "voluntad preventiva”. Fundamentado en los principios de los modernos sistemas sanitarios (universalidad, solidaridad y equidad), el Estado dispuso los recursos económicos necesarios en los Presupuestos Generales, aumentándolos de manera progresiva: de 6.619 .300 pesetas en el año 1920 se pasó a 9.990 .082 pesetas en 1931, 15.582.807 pesetas en 1932 y llegando a doblar la cantidad al año siguiente con una partida de 31.432.690 pesetas (Huertas, 2000; Castro-Molina, 2015).

Tanto las infraestructuras sanitarias como los recursos humanos no eran los adecuados. Los primeros habías quedado obsoletos y eran inapropiados para su finalidad; los segundos, precisaban de una reorientación en la que se definieran sus funciones y en la que se diera el protagonismo merecido tanto a la docencia como a la investigación, campos que habían quedado en un segundo plano (se da un marcado énfasis a la formación de los profesionales sanitarios basados en un cientificismo imperante en toda Europa, corriente que costó considerablemente instalar en nuestro país). La Dirección General de Sanidad creó nuevas secciones técnicas con el ánimo de poner en funcionamiento su proyecto: la Secretaria General Técnica, la Sección de Ingeniería y Arquitectura Sanitaria, la Sección de Higiene Social y Propaganda, la Sección de Higiene de la Alimentación, la Comisión Permanente de Investigaciones Sanitarias, la Sección de Higiene Infantil, el Departamento de Mortalidad materna, Morti-natalidad e Higiene Prenatal y Preescolar y, finalmente, la Sección de Psiquiatría e Higiene Mental (Castro-Molina, 2013; Huertas, 2000). 
Imagen 1: Sala de partos del Hospital Civil de la Beneficencia de Santa Cruz de Tenerife (años 30 del siglo $\mathrm{XX}$ )

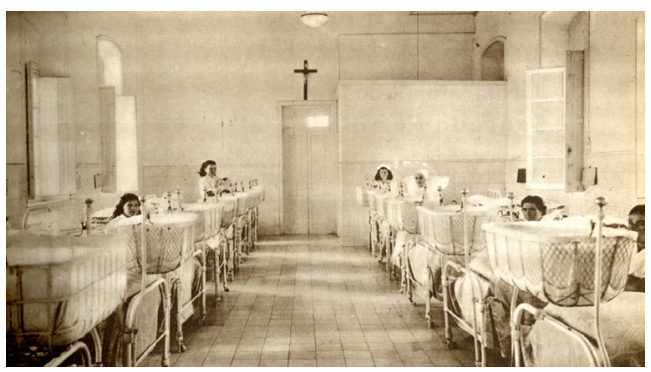

En 1934, con la Ley de Bases de Régimen Sanitario y la posterior Orden Ministerial de 13 de febrero de 1936 relacionados con la creación de un sistema sanitario, se enfatizó la intervención estatal en la organización de los servicios sanitarios locales, además de la reestructuración de los "desconocidos" centros primarios y secundarios de Higiene Rural. Ambos textos normativos buscaban la constitución de una red asistencial sanitaria, en la que se primara la prevención, la educación para la salud, la curación, la rehabilitación y la reinserción del individuo. Para lograr su objetivo, se dispusieron dispositivos sanitarios como los Centros Primarios y Secundarios de Higiene Rural (reorganizados y con una nueva interpretación), los Servicios de Higiene Infantil dependientes de los Institutos Provinciales de Higiene, los dispensarios móviles de Higiene Infantil, los dispensarios y sanatorios para la lucha antituberculosa-antivenérea, la Escuela Nacional de Sanidad, así como la creación de una Escuela Nacional de Enfermeras Visitadoras (García 2012; Alberdi, 2016).

Este último, el proceso de formación de las enfermeras y de los practicantes, desde un primer momento se vio mediatizado por todos aquellos cambios que progresivamente se fueron incorporando (Segura, 2013; Castro-
Molina, 2012). La intención en este área se pivotaba a partir de la profesionalización e institucionalización de este colectivo. La cualificación de la Enfermera no fue un proceso fácil. Técnicamente, se había logrado una ampliación del saber y de lo concerniente a su campo profesional de actuación. Hasta la fecha, la piedra angular profesional había girado en torno a unos "cuidados instintivos y de carácter natural", donde género y religión, en este caso la Católica, catalizaban cualquier actividad asistencial. Existió en todo momento una preocupación considerable por la cuerpo del conocimiento y la formación de las Enfermeras. Fruto de ello fueron los numerosos textos editados, que no solo aportaban el "saber preciso", sino que establecían y remarcaban las funciones de éstas. Aglutinaban materias como la Anatomía, la Fisiología y la Patología, además de abordar temas como la Higiene, las Técnicas Médicas, la Química, la Farmacología, la Dietética, la Fisioterapia y la Puericultura. Junto a ellas, las exigencias ético-morales que en ese preciso momento constituían un requisito para ser una "buena enfermera", entre las que se encontraban la bondad, la afectividad, la fortaleza, la paciencia, la puntualidad, la evidencia, el sacrificio y sobre todo, el secreto profesional (García, 2012; Segura, 2013).

Esta nueva forma de ver la profesión enfermera progresivamente fue dando sus frutos. La mutación del proceso formativo unido a la consolidación de la figura de las cuidadoras favoreció el avance hacia lugares nunca imaginados. Canarias no fue ajena a esta situación. En 1936, el consejero y médico del Excelentísimo Cabildo Insular de Tenerife, D. Manuel Bethencourt del Río, propuso un nuevo reglamento para el Hospital de Nuestra Señora de los Desamparados, posteriormente denominado Hospital Civil, con la pretensión de reorga- 
nizar los departamentos y la estructura orgánica interna, así como las funciones de cada una de las figuras de los profesionales que desempeñaban su labor en él. Primeramente, se estableció la creación de 6 plazas de practicantes numerarios y 2 supernumerarios, que debían distribuirse en el nosocomio equitativamente: 2 en Cirugía general (uno en cada servicio); 1 para Medicina Interna, Gastroenterología, Nutrición y Endocrinología; 1 para Tuberculosis, Enfermedades Infecciosas, Desinfección y Desinsectación; 1 en Ginecología, Urología y Dermatosifiliografía; y por último, 1 en Otorrino, Oftalmología, Odontoestomatología, Pediatría y Radioelectrología. La oposición era la única puerta de entrada a esta categoría, cuyo proceso contaba de un tribunal formado por el Director del Hospital, 3 Jefes de Servicio numerarios y 1 Practicante numerario que actuaba de Secretario. La prueba estaba formada por 2 ejercicios, el primero práctico eliminatorio, y el segundo teórico. Éste último, exigía la resolución de 3 de los 50 temas consignados en tan solo media hora (ACIT, caja 7088; Rodríguez, 2012).

Imagen 2: Expediente del Reglamento que establece la primera Directora de Enfermera.

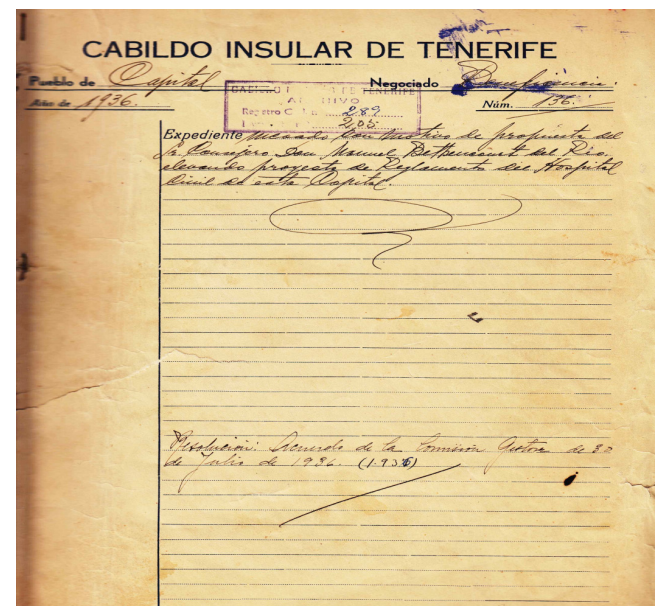

El citado reglamento, establecía además, las funciones del Practicante, articuladas en todo momento en el auxilio del médico y del cumplimento de sus prescripciones. A ellas se les unía el encargo del control y gestión de la Farmacia, la ejecución de intervenciones quirúrgicas de menor calado, las necroscopias y la gestión de las altas hospitalarias. De igual manera, cualquier candidata a ocupar una plaza de Enfermera debía pasar un proceso selectivo. El requisito principal para acceder a la prueba evaluatoria era poseer la titulación con validez oficial. Una vez superada el ejercicio, la seleccionada podía optar por una actividad laboral con carácter interno o externo (ACIT, caja 7088).

La nueva organización del Hospital insular modificaba el organigrama previo. El cuerpo de las Enfermeras estaba conformado por 1 Directora de Enfermeras, 1 Enfermera Jefe para cada servicio del Hospital y tantas Enfermeras como se precisara para atender a los enfermos ingresados. Para ser Directora de Enfermeras, la candidata debía ser titulada con preparación superior a la de las Enfermeras Jefes de Servicio. Ésta, la Directora, pertenecía a los miembro de la Junta Rectora, situación inédita hasta la fecha, y además se encargaba de velar por el cumplimiento del reglamento interno de Hospital en lo referente a su área de gestión y al cuidado de los enfermos. Incluso, coordinada tanto con el Director como con la Junta Rectora, era la responsable de confeccionar el horario y la distribución de las Enfermeras (cuadrantes), y de ser el referente de los médicos para la resolución de conflictos (ACIT, caja 7088; Rodríguez, 2012). Jerárquicamente, las diferentes Enfermeras Jefe existentes en las dependencias hospitalarias estaban bajo el mando de la Directora de Enfermeras. Tenían el encargo de la gestión tanto de las salas como 
de las Enfermeras, las alumnas de Enfermeras que recibían su formación y las Enfermeras Subalternas. Estas últimas, las Enfermeras Subalternas, estaban responsabilizadas de la limpieza de la cocina, del orden y organización de la ropería, de la actividad propia de los lavaderos y la limpieza general del Hospital (ACIT, caja 6545-7088; Rodríguez, 2012).

\section{DISCUSIÓN-CONCLUSIÓN}

Desconocer el pasado, lo que ya sucedió, obliga a que se repitan situaciones semejantes. Pero conocer la Historia, y en este caso, la de nuestra profesión, nos ayuda a tener argumentos para construir y consolidar nuestro futuro. La Enfermería, a diferencias de otras muchas profesiones, posee un camino breve en lo referente a su actividad laboral, lo que hace que carezca del reconocimiento social que se merece. Esto da pie a que los historiadores de la Enfermería prosigamos realizando descubrimientos en torno a ésta, que consolidan el cuerpo de la disciplina y permiten hacernos conscientes del camino ya hecho. Algunos autores han establecido dentro de nuestra historia dos "momentos" que han marcado un considerable avance de la Enfermería en España, la creación de las Consultas de Enfermería, que facilitaron la relación enfermera-usuario estableciendo una autonomía superior a la que estuvo presente durante la II República Española, y la creación de la figura de la Dirección de Enfermería, con iguales competencias que la Dirección Médica o la de Gestión, favoreciendo así el "autogobierno". Esto último momento, siempre ha sido ubicada cronológicamente en la década de finales de los ochenta del siglo XX (Hernández, 1996; Castro-Molina, 2016), pero es tras el hallazgo realizado en la isla de Tenerife, expuesto en el presente trabajo de investigación, el que deja claro que la historia actual difiere considerablemente de la "real". El reglamento estudiado establece el primer momento en el que se crea la figura de la Directora de Enfermeras, coincidente con el "ocaso republicano", facultándola y atribuyéndola con semejantes "poderes" que los adquiridos tras la Constitución de 1978, igualando su estatus al de las otras direcciones que tradicionalmente se han considerado capacitadas para su autogestión.

\section{FUENTES}

- Archivo del Cabildo Insular de Tenerife (ACIT). Sección de Beneficencia, caja 6544, 6545, 6546, 7088.

\section{BIBLIOGRAFÍA}

- Alberdi Castell, R.M. (2016). Retrospectiva. Una reflexión sobre la profesión enfermera y el trabajo comunitario en España desde los inicios hasta 1994. Madrid: Asociación Nacional de Enfermería de Salud Mental (ANESM), pp. 91-115.

- Castro Molina, F.J., Rodríguez Gómez, J.A. (2012). Uniformes e imagen social de los cuidadores profesionales. Santa Cruz de Tenerife: Colegio Oficial de Enfermeros de Santa Cruz de Tenerife.

- Castro-Molina, F.J. (2013). Arquitectura, asistencia y cui-dados. Manicomio Provincial de Tenerife. Santa Cruz de Tenerife: Colegio Oficial de Santa Cruz de Tenerife, pp.

38-42.

- Castro-Molina, F.J. (2015). Enfermería: ¿De dónde veni-mos? ¿Quiénes somos? ¿A dónde vamos?. Revista Pre-sencia, 11(21). Recuperado en http://www.indexf.com/presencia/n21/p10502.php.

- Castro-Molina, F.J. (2016). La enfermera victoriana: género e imperio. Cultura de los Cuidados (Edición digital), 20 (46). Recuperado en http://dx.doi.org/10.14198/ cuid .2016.46.09

- García Castillo, N. (2012).Un análisis educativo de la formación enfermera en España durante la Segunda República, de J.A. Vera Pérez y J.M. Hernández Conesa [comentario de texto]. Temperamentvm, 16. Recuperado en http://www.index-f.com/temperamentum/tn16/ t1602.php.

- Hernández Martín, F. (1996). Historia de la Enfermería en España: desde la Antigüedad hasta nuestros días. Madrid: Síntesis.

- Herrera Rodríguez, F. (2003). Notas sobre la Historia de la Enfermería en España (1977-2002). Llull, volumen 26, 157- 


\section{ᄃultura de las Cuidados}

- Huertas, R. (2000). Política Sanitaria: De la Dictadura de Primo de Rivera a la II República. Revista Especial de Salud Pública, 74, 2000, pp. 35-43.

- Matesanz Santiago, M.A. (2009).Pasado, presente y futuro de la Enfermería: una aptitud constante. Revista Adminis-tración Sanitaria, 7(2):243-60. Disponible en http://www.elsevier.es/es-revista-revistaadministracion-sa-nitaria-siglo-xxi-261-articulopasado-presente-futuro-enfermeria-una-13139761? redirectNew=true\#affa $>$ [Consultado el 10 de junio de 2016].
- Rodríguez Gómez, J.A., Ochoa Díez, L., Castro Molina, F.J. (2012). Centenario del Ilustre Colegio Oficial de Enfermería de Santa Cruz de Tenerife (1912-2012). Santa Cruz de Tenerife: Colegio Oficial de Santa Cruz de Tenerife.

- Segura López, G., Hernández Conesa, JM., Beneit Montesinos, J.V. ( 2013). Los sistemas formativos enfermeros durante la guerra civil Española (1936-1939). Arte y ciencia de la Sanación. Historia de la educación. Murcia: Diego Marín.

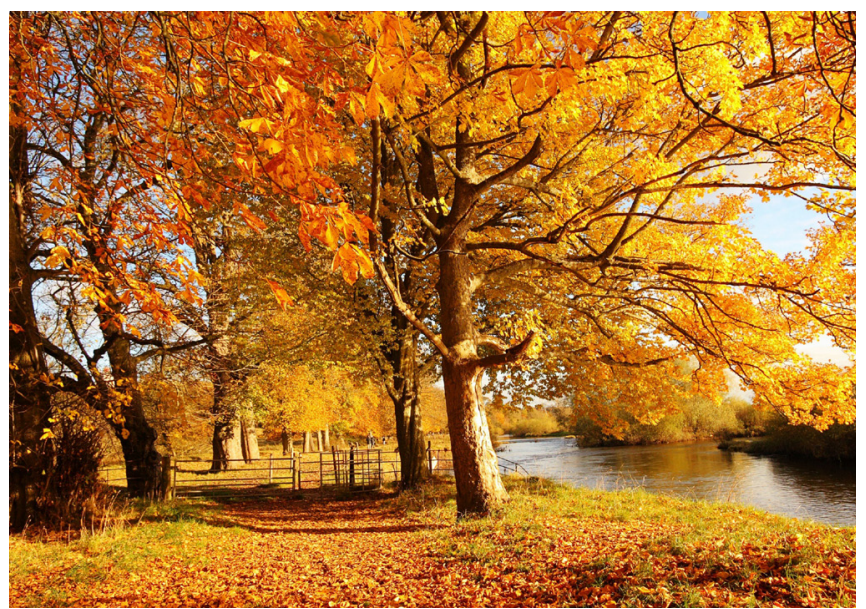

